Memorias del VII Encuentro Nacional de Experiencias en la Enseñanza de la Biología y la Educación Ambiental y II Congreso Nacional de Investigación en la Enseñanza de la Biología

\title{
LA NATURALEZA PROVEEDORA DE BIENESTAR Y BELLEZA, UN DISCURSO DESDE LA PERSPECTIVA LLANERA QUE ENRIQUECE LA CLASE DE CIENCIAS.
}

\begin{abstract}
THE NATURE PROVIDER OF WELL-BEING AND BEAUTY, A SPEECH FROM THE LLANERA PERSPECTIVE THAT ENRICHES THE CLASS OF SCIENCES.
\end{abstract}

\section{Andrés Arturo Venegas Segura ${ }^{1}$}

\section{Resumen}

Se presenta el discurso de Flor sobre la naturaleza, el cual resalta valores éticos, estéticos, emocionales, regulatorios enmarcados por un Ethos Llanero, donde el mundo natural es concebido como bello y ofrece utilidad, por tanto se debe respetar y conservar. Se utilizan los conglomerados de relevancias como dispositivo interpretativo de una carta al extraterrestre, un dibujo y una entrevista semi-estructurada, instrumentos que permitieron la recolección de la información. Palabras Clave: Idea de Naturaleza, conglomerados de relevancia, clase de ciencias naturales, Ethos Llaneros.

\section{Abstract.}

Is presented the Flor discourse on the nature, which highlights ethical, aesthetic, emotional, regulatory framed by a Ranger Ethos, where the natural world is conceived as beautiful and offers useful, therefore must be respected and preserved. Clusters are used as a device interpretative relevancies a letter to the alien, a drawing and a semi-structured instrument that allowed the collection of information.

Keywords: Idea of Nature, clusters of relevance, natural science class, Ethos Llaneros.

\section{Introducción}

Tomando como referencia la variedad de perspectivas que se tienen hoy en día frente al mundo natural en los diferentes ámbitos escolares, específicamente en la clase de ciencias naturales, se pretende evidenciar las posturas y los criterios de valor que orientan las ideas de naturaleza, a fin de entablar un motor de reconocimiento e identificación de su importancia en el universo discursivo, lo cual se configura como un elemento de reflexión pedagógica y didáctica en escenarios escolares heterogéneos. ${ }^{1}$ cDr. en Educación (Universidad Distrital). Esp. En Bioingeniería (Universidad Distrital). Lic en Física
(Universidad Distrital) y
Antropólogo (Universidad Nacional). aavenegas@udistrital.edu.co Doctorado Interinstitucional En Educación. Intercitec 
Memorias del VII Encuentro Nacional de Experiencias en la Enseñanza de la Biología y la Educación Ambiental y II Congreso Nacional de Investigación en la

\section{Enseñanza de la Biología}

De esta manera, el presente artículo tiene como objeto dar a conocer el discurso de Flor sobre la naturaleza y las interpretaciones emergentes a partir de la aplicación delos conglomerados de relevancias (Molina, 2012, 2000). Cabe resaltar que el discurso de Flor emerge en los diálogos entablados con ella a través de tres instrumentos de recolección de información. entrevista semiestructurada, una carta al extraterrestre, y un dibujo sobre la naturaleza.

Ésta interpretación es un resultado de la Tesis Doctoral "Ideas de Naturaleza de Niños y Niñas Sikuani y Llaneros de la Clase de Ciencias del Cuarto Grado de Básica Primaria del Colegio Agropecuario Silvino Caro Heredia en el Corregimiento del Viento en Vichada", dirigida por la Doctora Adela Molina.

La investigación se llevó a cabo en la clase de ciencias del cuarto grado en el Colegio Silvino Caro Heredia, departamento del Vichada, escenario con alta diversidad cultural, donde se encuentran niños y niñas con ascendencia Sikuani, Piapoco, Llaneros y Colonos. Por lo anterior, se asume que se trata de una clase de ciencias en la cual coexisten valores y creencias culturalmente diferenciadas, donde pueden existir negociaciones y traspasos de fronteras culturales. (Aikenhead, 2001, 1997, 1996; Cobern \& Loving 2001; Cobern, 1996a, 1996b, 1994, 1991; Kawasaki, 1997, 1996; Molina, 2012, 2002, 2000; Molina et al, 2006).

\section{Referente Conceptual Y Metodología}

Molina $(2000,2004)$ propone el concepto de Conglomerado de Relevancias, que estudia las interrelaciones entre conocimiento y cultura, postulando que el significado es dependiente del contexto y que la significación de la experiencia se puede llevar al lenguaje, el cual evidencia los valores (Ricoeur, 2006), las creencias, y aquello que es significativo.

Este dispositivo interpretativo se plantea con el fin de caracterizar las visiones y perspectivas sobre el mundo natural resultantes de los intercambios culturales en escenarios de alta diversidad cultural. Se basa en la idea de valor como decisión de Ricoeur, que permite resolver las opciones de significar presentes en los intercambios entre culturas (Molina, 2000, 2004).

La metodología de la investigación se enmarcó dentro de un enfoque cualitativo que permitió una aproximación al universo interpretativo del otro, los estudiantes.

De esta forma, la interpretación de sus ideas sobre la naturaleza se realizó a partir de los instrumentos mencionados y el relevamiento documental, este último basado en la metodología de análisis de documentos pues los textos utilizados se clasificaron, analizó y explicaron con la función de comprender el contexto cultural de Flory sus afirmaciones. Los documentos fueron fichados con base en los argumentos dados por los diferentes autores para describir los contextos culturales de las afirmaciones de la niña, lo cual es útil para referir las diversas 
Bio-grafia Escritos sobre la Biologia y su Enseñanza.

Edición Extra-Ordinaria. ISSN 2027-1034 P. p 630-637

Memorias del VII Encuentro Nacional de Experiencias en la Enseñanza de la Biología y la Educación Ambiental y II Congreso Nacional de Investigación en la Enseñanza de la Biología

ideas que expone, permitiendo el acercamiento y alejamiento a planteamientos sobre la naturaleza.

\section{Resultados E Interpretación.}

Flor es una niña que se identifica como llanera, para el año 2009 tiene 11 años de edad y se encuentra en cuarto de primaria, vive en el pueblo y su tiempo lo distribuye en actividades como estudiar, jugar, sembrar y cabalgar, las cuales disfruta y le generan felicidad. Su madre quien también se identifica como llanera, es parte fundamental en su formación, pues Flor manifiesta que su mamá es la persona que más sabe sobre la naturaleza. Su padre también es llanero, con él disfruta y aprende sobre las prácticas ganaderas al cabalgar y pastorear el ganado. En la escuela comparte con sus compañeros y maestros, quienes en muchos casos son indígenas de ascendencia Sikuani y Piapoco.

De esta manera, Flor se encuentra inmersa en una región que le permite generar múltiples lazos de pertenencia, dando forma y matices propios y únicos a su idea de la naturaleza y a su cosmovisión del mundo, pues se evidencia una convergencia de saberes tanto llaneros como indígenas. Ella demuestra conocimientos en torno a la siembra, la recolección, la ganadería y la manufactura, lo cual permite comprender algunos de sus planteamientos, que dan cuenta de una visión estética frente a la naturaleza. Además, se infiere que el "ser llanera" implica conocer y enseñar sobre los llanos orientales, sus condiciones de vida, sus prácticas socioculturales y exaltar la confluencia de múltiples saberes tanto indígenas, occidentales, como llaneros y escolares.

Al hablar del mundo natural como de quienes lo conforman (animales, frutos, flores, arboles, entre otros) se siente la disposición para mostrar y dejar un mensaje de cuidado y respeto hacia ella, exaltando además su belleza.

LOS CONGLOMERADOS DE RELEVANCIA: La interpretación del discurso de Flor se realizó teniendo en cuenta los conglomerados de relevancias los cuales ostentan unas jerarquías constituidas entre los criterios de valor, utilidad, naturalista, espacial, E-E-E (Ético, estético y emocional). Dichas jerarquías se evidencian en el gráfico uno (1) y se describen a continuación, 
Edición Extra-Ordinaria. ISSN 2027-1034 P. p 630-637

Memorias del VII Encuentro Nacional de Experiencias en la Enseñanza de la Biología y la Educación Ambiental y II Congreso Nacional de Investigación en la Enseñanza de la Biología

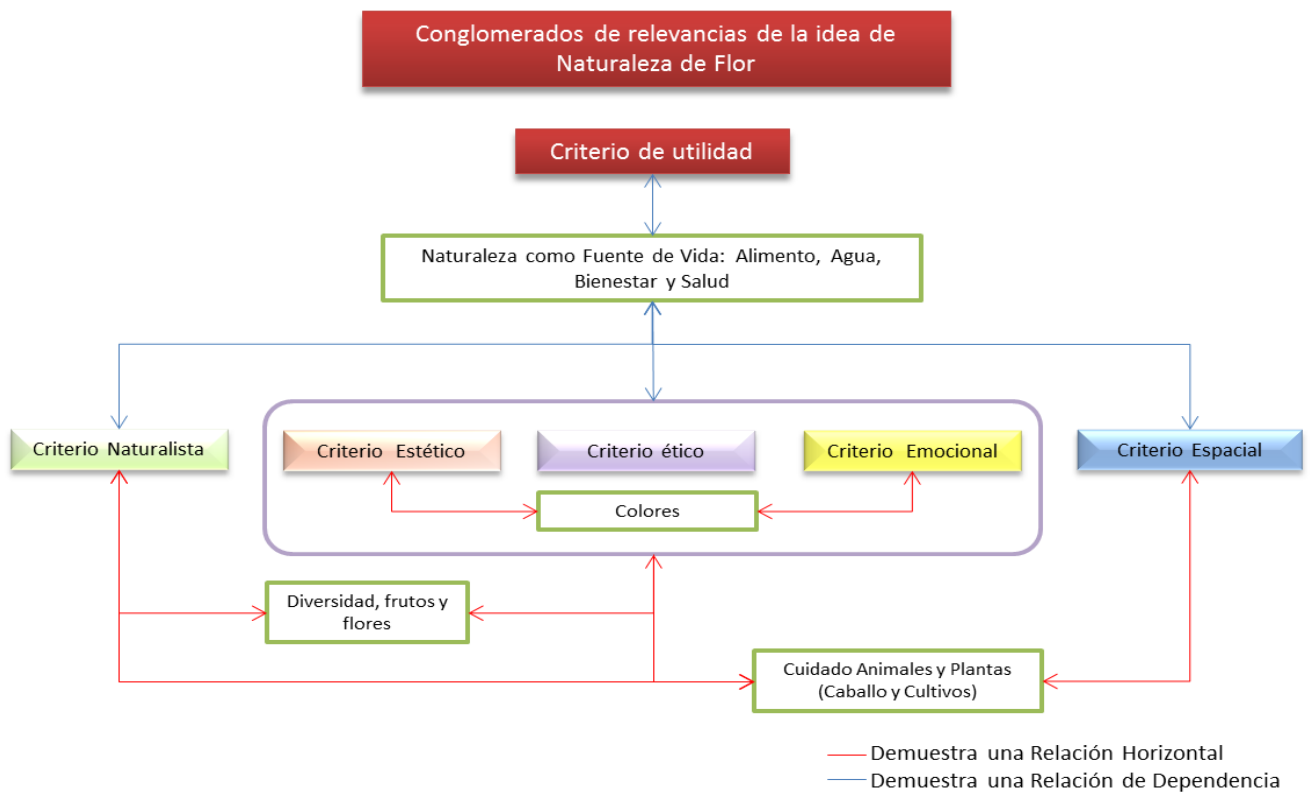

Gráfico 1. Jerarquías en el Conglomerado de Relevancias para Flor

- Jerarquía de dependencia: Configurada entre el criterio de utilidad con los criterios E-E-E, naturalista y espacial. La naturaleza para Flor se concibe como una fuente de vida que provee los elementos y recursos necesarios para la misma, el alimento, el agua, y todo aquello que es esencial para su bienestar y su salud. El criterio de utilidad regula los demás criterios dando paso a la conformación de la jerarquización de dependencia, sin llegar a ser utilitarista.

- Jerarquía Horizontal 1: Configurada entre los criterios E-E-E y espacial. Se evidencia la importancia que Flor confiere al cuidado de animales y plantas, a fin de mantener sus cualidades estéticas (belleza, fuerza, agilidad, rapidez, tamaño, colores, olores, formas, texturas, entre otras); teniendo en cuenta que estas pueden generar múltiples experiencias y emociones. Para ello, Flor reconoce la necesidad de adecuar ciertos espacios específicos para proteger y cuidar a los animales, revelando una posición ética con respecto a lo natural en relación con lo espacial.

- Jerarquía Horizontal 2: Configurada entre los criterios E-E-E y naturalista. La diversidad de seres está ligada tanto a su apreciación estética como a la descripción naturalista, así, se enfatiza en las características propias de las flores y los árboles, entre ellas su color. En cuanto a las frutas, Flor tiene en cuenta su textura, olor y sabor, aspectos que le suscitan emociones. Además se encuentra una actitud de cuidado hacia las características pues proporcionan la vida. 
Memorias del VII Encuentro Nacional de Experiencias en la Enseñanza de la Biología y la Educación Ambiental y II Congreso Nacional de Investigación en la Enseñanza de la Biología

Éstos criterios de valor permiten a Flor constituir su idea de naturaleza, con una mayor determinación del criterio de utilidad, el cual, orienta una serie de actuaciones, prácticas, acciones, emociones, posiciones y pensamientos particulares que vinculan de modo directo los demás criterios (E-E-E, naturalista y espacial). En este sentido, los criterios enunciados facilitan el reconocimiento y comprensión de la idea de naturaleza de Flor. Cabe resaltar, que el criterio E-E-E con respecto a la naturaleza, no le deja adoptar una posición utilitarista instalándola en una perspectiva orientada hacia el criterio de utilidad pues existe una regulación ética fundamentada en las características que para Flor hacen de lo natural algo bello, articulando de esta forma consideraciones de igualdad, respeto, responsabilidad y regulación.

De este modo, la naturaleza para Flor es una fuente de vida que provee alimento, agua, respiración, bienestar, salud y materias primas para construir un sinnúmero de elementos que le permiten la subsistencia en la Orinoquia.

En consecuencia, el criterio de utilidad encuentra una fuerte convergencia con afirmaciones tales como: "nosda la respiración..." (Flor: Carta); "Las frutas sirven para darnos agua (...) para darnos aire y las frutas son muy inportantes por que nos dan de comer nosdan bida nodan salud fortaleza y además son ricos sabrosos..." (Flor: Carta); "el árbol sirve para sacar madera como para aser cosas pa aser pupitres, mesas, camas..." (Flor: Carta).

Como se advierte en estas enunciaciones, la naturaleza provee alimento por medio de las frutas, abastece de madera para realizar herramientas y además proporciona la respiración. En este sentido, la idea de naturaleza de Flor evidencia un elemento vital pues ésta es quien da.

Ampliando esta interpretación, se observa como Flor considera los árbolesproveedores de materia prima para elaborar elementos empleados por el hombre en su vida cotidiana como el pupitre de la escuela, las mesas y camas de su casa, "... el árbol sirve para sacar madera como para aser cosas pa aser pupitres, mesas, camas..." (Flor: Carta).

Por otra parte, las plantas se constituyen como la fuente principal de alimento, de tal forma, prácticas como la recolección, la ganadería y la siembra son actividades que le permiten alcanzar el bienestar, de ahí el criterio de utilidad y la jerarquía de dependencia con los demás criterios. En este sentido, las plantas por medio de sus frutos otorgan bienestar y vida a los seres humanos, pues brindan alimento, agua y aire:"...Las frutas sirven para darnos agua alimento para darnos aire y las frutas son muy inportantes por que nos dan de comer" (Flor: Carta). Ello confiere 
Memorias del VII Encuentro Nacional de Experiencias en la Enseñanza de la Biología y la Educación Ambiental y II Congreso Nacional de Investigación en la Enseñanza de la Biología

salud y fortaleza, características que entran en concordancia nuevamente con el criterio de utilidad, "nosdan bida nodan salud fortaleza..." (Flor: Carta).

Es preciso mencionar que este criterio de utilidad converge con el criterio $E-E-E$, en especial con el emocional, constituyendo la jerarquía de dependencia, pues las frutas proporcionan alimento y ocasionan deleite y satisfacción al ser ingeridas, “...y además son ricos sabrosos" (Flor: Carta).

De acuerdo con lo anterior, Flor expone una variedad de frutas, "Investigador: ¿Qué clase de frutas (...) conoces? / 'Flor': (...) la papaya, el coco, el mango, la piña, la caña, (...) manzana, la pera, eh la uva” (Flor: Entrevista) demostrando cómo el criterio de utilidad está ligado al naturalista al dar cuenta de la diversidad de frutos, exhibiendo nuevamente una relación jerárquica de dependencia.

Es importante resaltar que para Flor hay restricciones con respecto al consumo de carne de algunos animales, característica opuesta a la situación con las plantas, no obstante, tanto plantas como animales le brindan bienestar alimentario, “...Investigador: ¿Todos animales se pueden comer? / 'Flor': No / Investigador: ¿Cuáles no? / 'Flor': El león, el perro...” (Flor: Entrevista).

El caballo es representativo para Florpues es su dibujo sobre la naturaleza (Véase Ilustración 1).Además, en la entrevista explica su importancia y el gusto que le suscita, "Investigador: ¿Te gustan los caballos? / 'Flor': Si”. El gusto por el caballo se asocia a la utilidad que le presta para realizar ciertas actividades, en especial, el cuatreo en la ganadería, "Investigador: ¿Por qué? / 'Flor': Pues pa' uno montarse. (...) vamos a correr ganado despacio porque da mucho medio (...) ahorita estamos en San ... [con] Mi papá, nos íbamos a correr a traer ganado..." (Flor: Entrevista).

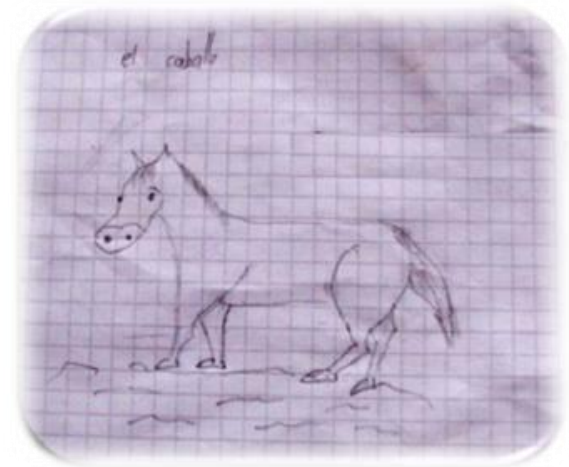

Ilustración 1. Dibujo sobre la naturaleza de Flor

Asimismo, Flor explica que el lugar de descanso del caballo y las vacas debe ser adecuado por los seres humanos debido a la labor que éstos animales 
Memorias del VII Encuentro Nacional de Experiencias en la Enseñanza de la Biología y la Educación Ambiental y II Congreso Nacional de Investigación en la Enseñanza de la Biología

desempeñan, de esta manera, la niña da cuenta del criterio espacial subordinado a la utilidad, "...Investigador: (...) ¿los caballos dónde viven? / 'Flor': Los caballos viven (...), en donde hay pasto pa' ellos comer (...) pa los caballos tienen que haber un lotecito y pa' las vacas también..." (Flor: Entrevista).

En este punto, Flor demuestra nuevamente la jerarquía de dependencia entre el criterio de utilidad, el naturalista, el espacial, y el E-E-E (en este caso emocional y ético), pues el caballo es un animal que le sirve a ella y a su familia para desarrollar actividades propias de la región, además de generarle gusto, de ahí que la relación utilidad-gusto implique tener cuidado al montar el caballo, lo cual le causa miedo o alegría, entre otras emociones.

Cabe resaltar para finalizar este apartado, que Flor considera el agua esencial para la vida, ya que sin ella no podrían existir las plantas, los animales y el ser humano, como comenta a lo largo de la entrevista. En concordancia, ratifica las plantas como eje central de su idea sobre la naturaleza, en especial las flores y los frutos. Asimismo, agua denota importancia para el crecimiento, sostenimiento y conservación de características físicas de las plantas como su color, en este sentido, vislumbra una relación entre el criterio E-E-E y el espacial, subordinados al de utilidad, mostrando una jerarquía de dependencia, "Investigador: ¿Y si no existiera el agua en el mundo que pasaría? / 'Flor': Se morirían las flores y nosotros también (...) Porque si no tenemos de donde vamos a tomar agua..." (Flor: Entrevista).

\section{A Manera De Conclusión.}

Las ideas de naturaleza más relevantes para Flor están marcadas por el Ethos Llanero,donde la clase de ciencias naturales proporciona algunos elementos para su discurso sin ser fundamentales o tener fuerza.

Para flor lanaturaleza es proveedora de bienestar ya que le brinda el alimento para su subsistencia, no obstante, al estar el criterio de utilidad ligado al criterio E-E-E, el discurso vislumbra una posición de cuidado hacia la naturaleza, en la cual están articuladas consideraciones de respeto, responsabilidad y regulación para con ella, al considerarla como fuente de vida, belleza y bienestar.

\section{Bibliografia.}

Aikenhead, G.S. (1996). Science education: Border crossing into the subculture of science. Studies in Science Education, 27, p. 1-52.

Aikenhead, G.S. (1997). Toward a First Nations cross-cultural science and technology curriculum. Science \& Education, 81, p. 217-238.

Aikenhead, G.S. (2001). Integrating Western And Aboriginal Sciences: CrossCultural Science Teaching. Research in Science Education, 31 (3), p. 337-355. 
Bio-grafia Escritos sobre la Biología y su Enseñanza.

Edición Extra-Ordinaria. ISSN 2027-1034 P. p 630-637

Memorias del VII Encuentro Nacional de Experiencias en la Enseñanza de la Biología y la Educación Ambiental y II Congreso Nacional de Investigación en la Enseñanza de la Biología

Cobern, W., \& Loving, C. (2001). Defining 'Science' in a Multicultural World: Implications for Science Education. Science \& Education, 85, p. 50-67.

Cobern, W. (1996a). Constructivism And Non-Western Science Education Research. International Journal of Science Education, 4(3), p. 287-302.

Cobern, W. (1996b). World View, theory and conceptual change in science education. Science Education International, 80(5), p. 579-610.

Cobern, W. (1994a). Alternative Constructions Of Science And Science Education. Southern Africa Association for Mathematics and Science Education Research, University of Durban-Westville, Durban, South Africa, p.27-30 January

Cobern, W. (1994b). World View, culture, and science education. Science Education International, 5(4), p. 5-8.

Cobern, W. (1991). World View Theory and Science Education Research. Manhattan-Kansas: NARST.

Kawasaki, K. (1997). An aspect of science education in Japan within the framework based on structural linguistics. In Ogawa, M. (Ed.), Effects of traditional cosmology on science education p. 48-62.

Kawasaki, K. (1996). The concepts of science in Japanese and Western education. Science \& Education, 5, p. 1-20

Molina, A. (2012). Contribuciones Metodológicas Para El Estudio De Relaciones Entre Contexto Cultural E Ideas Sobre La Naturaleza De Niños Y Niñas. Universidad Distrital Francisco José de Caldas. Cuadernos Número 9. P. 63-88

Molina, A., Mojica, L. \& López, D. (2006). Ideas de los niños y niñas sobre la naturaleza: estudio comparado. Revista Científica, 7. p. 41-62.

Molina, A. (2002). Conglomerado de relevancias y formación científica de niños, niñas y jóvenes. Revista Científica (4), p. 187-200.

Molina, A. (2000). Conhecimento, Cultura e Escola: Um estudo de suas Interrelações a partir das idéias dos alunos (8-12 anos) sobre os espinhos dos cactos.

Ricoeur, P. (2006). Teoría de la interpretación. Discurso y excedente de sentido. Ed. Siglo XXI. México. 